\title{
Variação geográfica em Corydoras paleatus (Jenyns) (Siluriformes, Callichthyidae) do sul do Brasil
}

\author{
Oscar A. Shibatta \& Ana C. Hoffmann
}

Museu de Zoologia, Departamento de Biologia Animal e Vegetal, Centro de Ciências Biológicas, Universidade Estadual de Londrina.86051-970 Londrina, Paraná, Brasil. E-mail: shibatta@uel.br

\begin{abstract}
Geographic variation in Corydoras paleatus (Jenyns) (Siluriformes, Callichthyidae) from southern Brazil. The species Corydoras paleatus (Jenyns, 1842) is widely distributed in Southern Brazil, occurring from Rio Grande do Sul to the north of Paraná State. Studies related to this species populations are considered important once this fish is distributed in a region with great anthropogenic interference and also because its economic importance, given that this species is commercialised as ornamental fish. In the present study sixteen morphometric characters and six meristic characters were used for morphological evaluation in populations of Tibagi and Iguaçu river basins (Paraná) and Lagoa dos Quadros (Rio Grande do Sul). In spite of the number of lateral plates being more variable in specimens from Iguaçu river, the overlap of plates and fin rays counts did not allow a safe discrimination of populations. However, principal components analysis allowed to differentiate populations by the allometric growth of the morphometric characters. Besides, the sample from rio Iguaçu was morphometrically discriminated of those from Tibagi river and Lagoa dos Quadros in the first canonical axis, while these two populations were separated in the second axis. The intraspecific variation, observed in this species, clearly shows that population morphometric analysis can be useful in the analysis of biological diversity of a given region. The morphometric differences and the geographical isolation are indicating that those populations are subject to different selective processes.

KEY WORDS. Allometry, biological diversity, multivariate morphometric, Neotropical fishes, small catfishes.
\end{abstract}

RESUMO. A espécie Corydoras paleatus (Jenyns, 1842) está amplamente distribuída na região Sul do Brasil, ocorrendo desde o Rio Grande do Sul até o norte do Paraná. Estudos populacionais nessa espécie são considerados importantes devido à sua distribuição em uma região com forte interferência ambiental humana e pelo interesse comercial, uma vez que é vendida como peixe ornamental. Dezesseis caracteres morfométricos e seis caracteres merísticos foram utilizados para avaliação morfológica das populações das bacias dos rios Tibagi e Iguaçu (Paraná) e Lagoa dos Quadros (Rio Grande do Sul). Apesar do número de placas laterais ser mais variável nos exemplares do rio Iguaçu, a sobreposição das contagens tanto das placas quanto dos raios das nadadeiras não permitiu a discriminação segura das populações. Entretanto, foi observado com a análise dos componentes principais, que essas podem ser diferenciadas pelo crescimento alométrico dos caracteres morfométricos. Além disso, a amostra do rio Iguaçu ficou discriminada morfometricamente do rio Tibagi e Lagoa dos Quadros no primeiro eixo canônico, enquanto estas ficaram separadas no segundo eixo. A variação intraespecífica, observada nessa espécie, evidencia que análises morfométricas populacionais podem ser úteis na análise da diversidade biológica de determinada região. As diferenças morfométricas, aliadas ao isolamento geográfico, são indícios de que essas populações estão sujeitas a diferentes processos seletivos.

PALAVRAS CHAVE. Alometria, diversidade biológica, morfometria multivariada, peixes neotropicais, pequenos bagres.

Corydoras Lacépède, 1803 é composto por 143 espécies (ReIs 2003) amplamente distribuídas na América do Sul. Corydoras paleatus (Jenyns, 1842) foi coletada pela primeira vez por Charles Darwin em localidade não indicada. A distribuição geográfica compreende a bacia do rio Paraná e rios costeiros do Brasil e Uruguai (ReIs 2003). No sul do Brasil, região com fortes interfe- rências antrópicas, está presente nos Estados do Paraná, Santa Catarina e Rio Grande do Sul (Fowler 1954, Garavello et al. 1997). É um peixe facilmente diferenciado de seus congêneres pelo padrão de colorido de três manchas castanho escuras nas regiões lateral e dorsal do tronco, e nadadeira caudal estriada. Além disso, machos têm a nadadeira dorsal alongada, com os

Revista Brasileira de Zoologia 22 (2): 366-371, junho 2005 
dois primeiros raios divididos maiores que o acúleo (Fig. 1). A espécie é de interesse comercial, sendo vendida como peixe ornamental (Burgess 1989).

Várias amostras foram obtidas nas bacias dos rios paranaenses Tibagi e Iguaçu e outra na Lagoa dos Quadros, extremo sul do Brasil, na porção leste do estado do Rio Grande do Sul. Este estudo foi realizado para avaliar a ocorrência de diferenças morfológicas diagnósticas nessas populações de C. paleatus em distintos sistemas de drenagens.

\section{MATERIAL E MÉTODOS}

As amostras analisadas de C. paleatus estão depositadas no Laboratório de Biologia de Peixes do Instituto de Biociências da Universidade Estadual Paulista, Botucatu, São Paulo (LBP), Museu de História Natural do Capão da Imbuia, Curitiba, Paraná (MCI), Museu de Ciências e Tecnologia da Pontifícia Universidade Católica do Rio Grande do Sul, Porto Alegre, Rio Grande do Sul (MCP), Museu de Zoologia da Universidade Estadual de Londrina, Londrina, Paraná (MZUEL) e Núcleo de Pesquisa em Limnologia, Ictiologia e Aqüicultura da Universidade Estadual de Maringá, Maringá, Paraná (NUPÉLIA). O seguinte material foi examinado: Brasil. Paraná: MZUEL 612, rio Lambari, Sapopema, 13/09/96 (Equipe de coleta de peixes da Universidade Estadual de Londrina (ECPUEL)), 6 de 7 exs. (35,95-46,80 mm CP); MZUEL 702, rio Tibagi, Telêmaco Borba, 14/04/95 (ECPUEL), 2 exs. (42,10-49,35 mm CP); MZUEL 820, rio Tibagi, Sapopema, 21/05/95 (ECPUEL), 6 exs. (36,45-53,40 mm CP); MZUEL 822, rio Tibagi, Telêmaco Borba, 08/08/90 (ECPUEL), 1 ex. (47,30 mm CP); MZUEL 825, rio Lambari, Sapopema, 25/08/ 94 (ECPUEL), 6 exs. (35,60-44,20 mm CP); MZUEL 1436, rio Tibagi, Limoeiro, 09/10/97 (ECPUEL), 2 exs. (49,05-51,00 mm CP); MZUEL 1476, rio Tibagi, 28/11/97 (ECPUEL), 1 ex. (43,45 mm CP); MZUEL 1541, rib. Água da Floresta, Jataizinho, 09/06/ 98 (ECPUEL), 1 ex. (35,37 mm CP); MZUEL 1562, rib. Barra Funda, 21/08/98 (ECPUEL), 1 ex. (37,60 mm CP); LBP 380, rio Iguaçu, reservatório de Caxias, estação de amostragem de Porto Vará, divisa entre os municípios de Três Barbas do Paraná e Nova Prata do Iguaçu, 13/12/1998, 19 de 27 exs. (24,75-45,55 mm CP); rio Iguaçu, 08/88 (M. Takase), 4 de 5 exs. (46,15-59,10 mm CP); Pq. Municipal do Iguaçu, 20/07/95 (S. Sasaoka, V.S. Abilhoa e M. Cordeiro), lote 71, 8 exs., (29,60-30,85 mm CP). Rio Grande do Sul: MCP 13633, Sanga afluente da Lagoa dos Quadros, 01/ 10/89 (S. O Kullander), 10 de 11 exs. (32,40-39,15 mm CP); LBP 323, Rio Grande, 5 exs. (33,35-45,00 mm CP) (Fig. 2).

Um total de 65 espécimes foi medido e utilizado para a contagem de número de placas e raios (10 de 16 exemplares da Lagoa dos Quadros, 18 de 36 de Tibagi e 31 exemplares de Iguaçu). As medidas foram tomadas ponto a ponto com um paquímetro com precisão de $0,05 \mathrm{~mm}$ sob um microscópio estereoscópico. Machos e fêmeas adultas foram identificados pelo comprimento da nadadeira dorsal, mas este caráter não foi mensurado devido aos danos nos raios moles ou por estes não estarem completamente desenvolvidos. Os seguintes caracteres foram obtidos: Comprimento padrão $(\mathrm{CP}$, da ponta do focinho em seu vértice até o último par de placas no pedúnculo caudal); comprimento da cabeça (CC, da ponta do focinho até a borda do opérculo em linha horizontal); distância pré-dorsal (DPD, da ponta do focinho até a base anterior da nadadeira dorsal); comprimento da base da dorsal (CBD, da margem anterior à posterior da base da nadadeira dorsal); distância pré-ventral (DPV, da ponta do focinho à base da nadadeira ventral); comprimento da base da anal (CBA, da margem anterior à posterior da base da nadadeira anal); altura do corpo (AC, em linha vertical em frente à nadadeira dorsal); altura do pedúnculo caudal (APC, a menor altura do pedúnculo); diâmetro do olho (DO, distância tomada horizontalmente entre as bordas dos olhos); distância interorbital (DIO, a menor distância dorsal entre os olhos); comprimento do focinho (CF, da ponta do focinho à borda anterior do olho esquerdo); comprimento do barbilhão maxilar (CBM, do meio da boca à extremidade do barbilhão maxilar); altura da cabeça (Acb, tomada em linha vertical à borda posterior do olho esquerdo); largura da cintura escapular (LCE, a maior distância entre um lado e outro do cleitro); distância entre a dorsal e adiposa (DDAD, tomada da base posterior da nadadeira dorsal até o início da nadadeira adiposa); altura do espinho da dorsal (AED, da base à extremidade do espinho da nadadeira dorsal).

Os seguintes caracteres merísticos (contagens) foram obtidos: Placas em linha longitudinal (total de placas); raios da dorsal (número total de raios menos o do mecanismo de trava); raios da peitoral (número total); raios da ventral (número total); raios da anal (número total); raios da caudal (somente os raios principais).

Os coeficientes de alometria multivariados de cada amostra foram calculados com a metodologia proposta por JOLICOUER (1963), a partir do primeiro autovetor da análise de componentes principais (ACP). Este eixo representa o tamanho quando todos os valores do autovetor são positivos (NefF \& MARCus 1980). O caráter com coeficiente maior que 1 foi considerado alometricamente positivo, menor que 1 alometricamente negativo e isométrico quando igual a 1 .

Os grupos foram analisados segundo protocolo de ReIs et al. (1990) para Análise das Variáveis Canônicas independentes de tamanho (AVC). Os programas "Shear" (MACLEOD 1990) e SAS (SAS 1985) foram usados para calcular a ACP e AVC, respectivamente.

\section{RESULTADOS}

A tabela I mostra sobreposição em todos os caracteres merísticos. Mesmo assim, o número de placas laterais foi o caráter que indicou diferença entre as amostras. A amostra do rio Iguaçu apresentou de 20 a 23 placas e número modal 22, enquanto o número de placas alcançou de 22 a 23, número modal 23, nas amostras do rio Tibagi e Lagoa dos Quadros (Tab. I). A amostra do rio Iguaçu também teve a maior variação em número de raios das nadadeiras. (Tab. I).

Revista Brasileira de Zoologia 22 (2): 366-371, junho 2005 


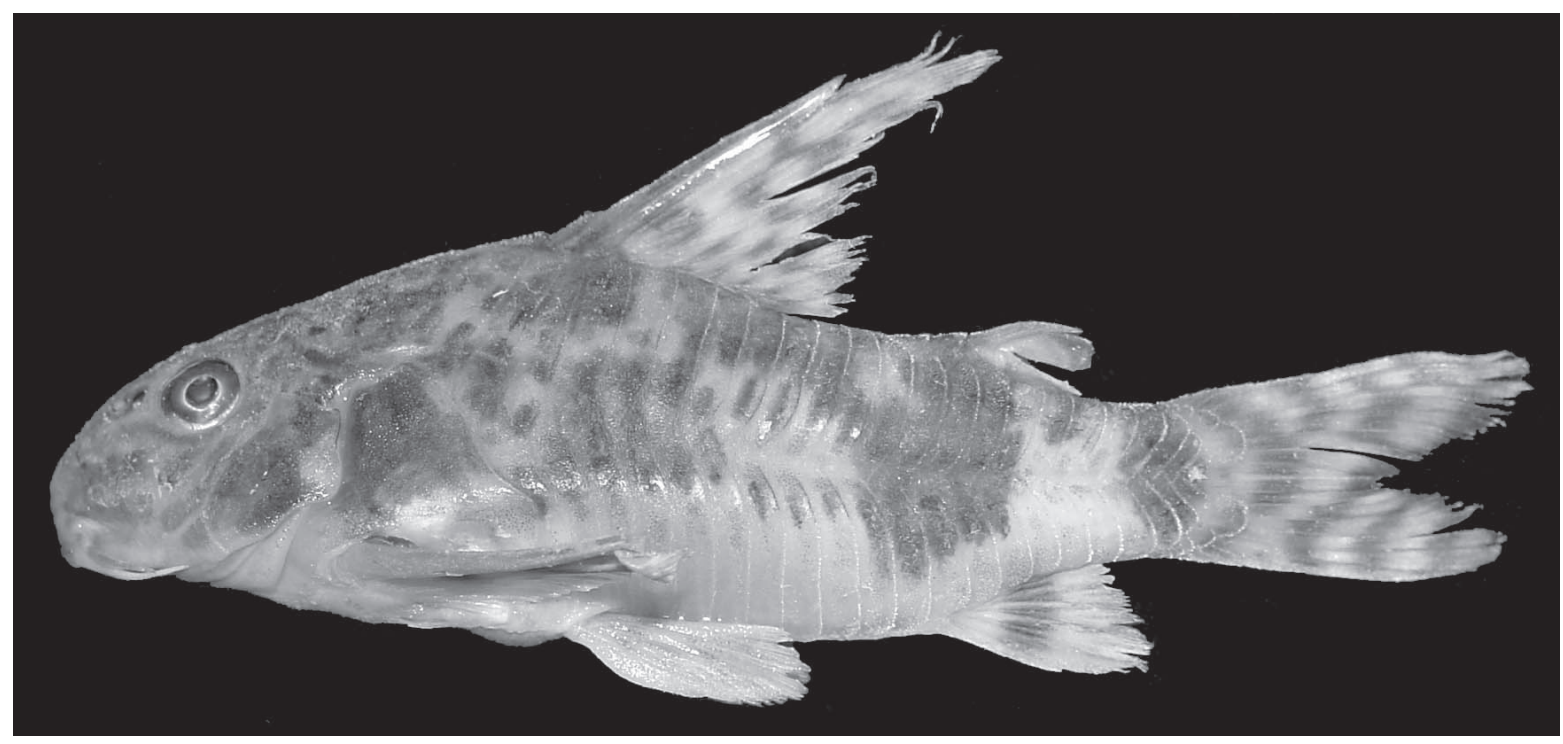

Figura 1. Exemplar macho de Corydoras paleatus da bacia do rio Tibagi (MZUEL 1718, 48,40 mm CP).

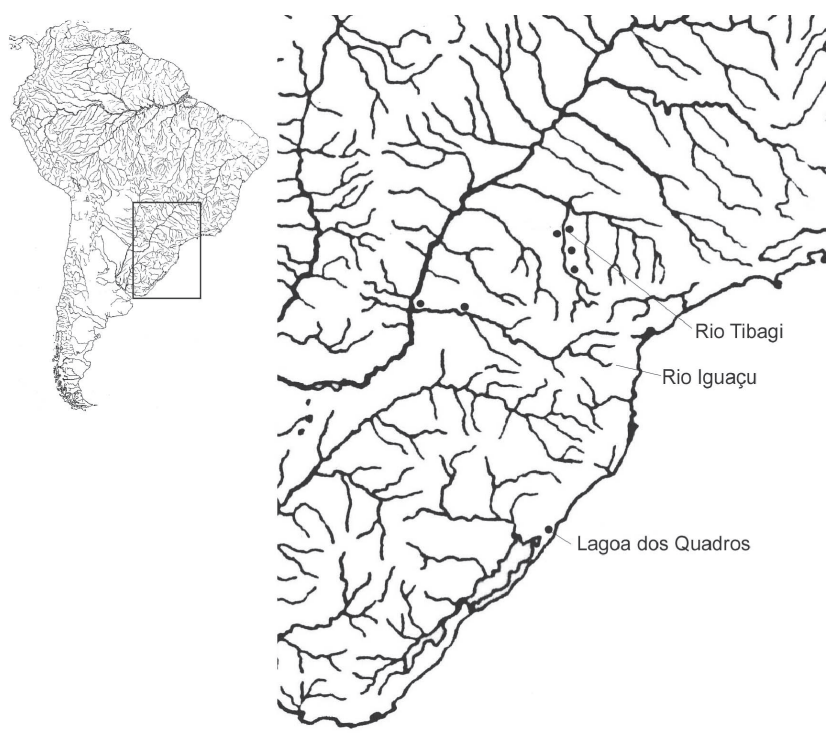

Figura 2. Mapa da América do Sul, com detalhe da região sul do Brasil apresentando as três bacias hidrográficas amostradas e os pontos de coleta.
O dimorfismo sexual não foi evidenciado com os caracteres analisados. O tamanho do acúleo da nadadeira dorsal é semelhante em ambos os sexos, apesar de ocorrer prolongamento da porção flexível do acúleo e do primeiro raio ramificado nos machos.

A ACP em cada amostra resultou em diferentes graus de representação da matriz original pelo primeiro eixo do componente principal, que pode ser interpretado como tamanho, pois todos os valores do autovetor foram positivos. O primeiro componente principal da população de Lagoa dos Quadros teve um baixo valor $(59,7 \%)$ significando que as medidas não foram proporcionais entre os indivíduos. A população do rio Tibagi teve um valor intermediário para o primeiro componente principal $(65,2 \%)$ e a população do rio Iguaçu o mais alto $(86,1 \%)$. Todas as populações apresentaram um crescimento alométrico negativo do comprimento da cabeça, distância prédorsal e distância pré-ventral. A população do rio Iguaçu apresentou quatro caracteres compartilhados com a população de Lagoa dos Quadros (três coeficientes alométricos positivos e um negativo) e cinco com a população de rio Tibagi (três coeficientes alométricos negativos e dois positivos). Tibagi por sua vez apresentou quatro caracteres compartilhados com Lagoa

Tabela I. Freqüência (porcentagem) dos caracteres merísticos em Corydoras paleatus do rio Tibagi $(n=17)$, rio Iguaçu $(n=32)$ e Lagoa dos Quadros $(n=16)$.

\begin{tabular}{|c|c|c|c|c|c|c|c|c|c|c|c|c|c|c|}
\hline \multirow[b]{2}{*}{ Merística } & \multirow[b]{2}{*}{20} & \multirow[b]{2}{*}{21} & \multirow[b]{2}{*}{22} & \multirow[t]{2}{*}{ Placas } & \multicolumn{2}{|c|}{ Raios da dorsal } & \multicolumn{2}{|c|}{ Raios da peitoral } & \multicolumn{2}{|c|}{ Raios da ventral } & \multicolumn{2}{|c|}{ Raios da anal } & \multicolumn{2}{|c|}{ Raios da caudal } \\
\hline & & & & & $1+7$ & $1+8$ & $1+7$ & $1+8$ & $\mathrm{i}+5$ & $\mathrm{i}+6$ & $\mathrm{i}+5$ & $\mathrm{i}+6$ & $\mathrm{i}+12+\mathrm{l}$ & $\mathrm{i}+13+\mathrm{i}$ \\
\hline Tibagi & 0 & 0 & 17,60 & 82,40 & 0 & 100 & 0 & 100 & 11,76 & 88,24 & 0 & 100 & 100 & 0 \\
\hline Iguaçu & 6,25 & 18,75 & 56,25 & 18,75 & 6,25 & 93,75 & 18,75 & 81,25 & 6,25 & 93,75 & 18,75 & 81,25 & 96,88 & 3,12 \\
\hline L. Quadros & 0 & 0 & 43,75 & 56,25 & 0 & 100 & 31,25 & 68,75 & 12,50 & 87,50 & 0 & 100 & 100 & 0 \\
\hline
\end{tabular}

Revista Brasileira de Zoologia 22 (2): 366-371, junho 2005 
dos Quadros (três coeficientes alométricos negativos e um positivo). A população do rio Tibagi apresentou alometria negativa para o comprimento padrão, comprimento da base da nadadeira dorsal e distância entre as nadadeiras dorsal e anal e alometria positiva para o comprimento do barbilhão maxilar. A população do rio Iguaçu apresentou crescimento isométrico na altura do corpo, alometria positiva no diâmetro do olho e largura da cintura escapular, e alometria negativa no comprimento do focinho. A população de Lagoa dos Quadros difere das demais pela alometria positiva no comprimento da base na anal e distância interorbital, crescimento isométrico na altura do pedúnculo caudal, e alométrico negativo na altura da cabeça e comprimento do espinho da dorsal (Tab. II).

Tabela II. Peso dos caracteres e coeficientes de alometria (CA) do primeiro componente principal (PC1) das populações analisadas de Corydoras paleatus.

\begin{tabular}{|c|c|c|c|c|c|c|}
\hline & \multicolumn{2}{|c|}{ Tibagi } & \multicolumn{2}{|c|}{ Iguaçu } & \multicolumn{2}{|c|}{ Lagoa dos Quadros } \\
\hline & PC1 & $C A$ & PC1 & $C A$ & PC1 & CA \\
\hline$C P$ & 0,20 & 0,80 & 0,27 & 1,08 & 0,26 & 1,04 \\
\hline $\mathrm{CC}$ & 0,20 & 0,80 & 0,21 & 0,84 & 0,21 & 0,84 \\
\hline DPD & 0,23 & 0,92 & 0,24 & 0,96 & 0,20 & 0,80 \\
\hline CBD & 0,22 & 0,88 & 0,29 & 1,16 & 0,43 & 1,72 \\
\hline DPV & 0,21 & 0,84 & 0,22 & 0,88 & 0,24 & 0,96 \\
\hline CBA & 0,12 & 0,48 & 0,16 & 0,64 & 0,30 & 1,20 \\
\hline$A C$ & 0,22 & 0,88 & 0,25 & 1,00 & 0,19 & 0,76 \\
\hline APC & 0,20 & 0,80 & 0,22 & 0,88 & 0,25 & 1,00 \\
\hline DO & 0,16 & 0,64 & 0,28 & 1,12 & 0,21 & 0,84 \\
\hline DIO & 0,22 & 0,88 & 0,21 & 0,84 & 0,30 & 1,20 \\
\hline $\mathrm{CF}$ & 0,26 & 1,04 & 0,16 & 0,64 & 0,31 & 1,24 \\
\hline CBM & 0,43 & 1,72 & 0,06 & 0,24 & 0,05 & 0,20 \\
\hline $\mathrm{ACb}$ & 0,42 & 1,68 & 0,32 & 1,28 & 0,17 & 0,68 \\
\hline LCE & 0,23 & 0,92 & 0,28 & 1,12 & 0,21 & 0,84 \\
\hline DDAd & 0,21 & 0,84 & 0,38 & 1,52 & 0,29 & 1,16 \\
\hline AED & 0,26 & 1,04 & 0,29 & 1,16 & 0,15 & 0,60 \\
\hline
\end{tabular}

Foram obtidos dois eixos canônicos com a AVC: o primeiro representou $57,4 \%$ da matriz original e o segundo $42,6 \%$. No primeiro eixo foi possível notar a discriminação da população do rio Iguaçu das populações do Tibagi e Lagoa dos Quadros, enquanto que estas se separaram no segundo eixo (Fig. 3). A população do Iguaçu ficou em posição intermediária às outras populações neste eixo. A população do Iguaçu apresentou como caracteres diagnósticos os maiores comprimentos da base da nadadeira dorsal, do focinho, da cabeça e menores a altura do pedúnculo caudal, diâmetro do olho e comprimento da base da nadadeira anal. A população do rio Tibagi foi distinguida de Lagoa dos Quadros pelos maiores valores da distância pré-ventral, do comprimento do focinho e da distância

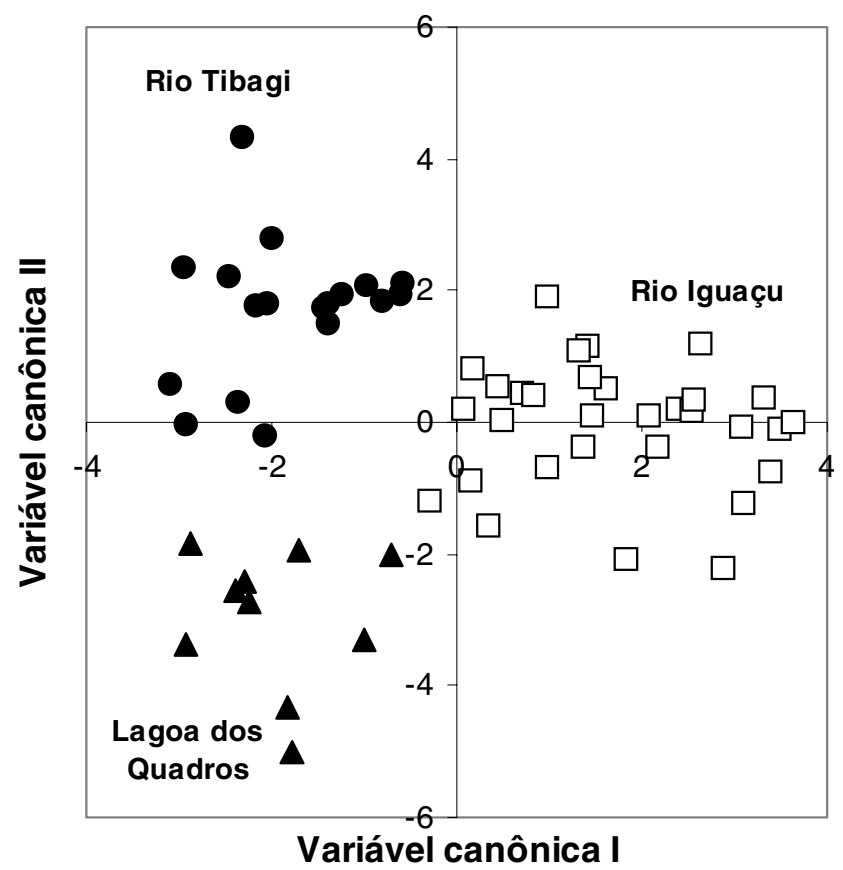

Figura 3. Gráfico de dispersão dos escores individuais das amostras combinadas de Corydoras paleatus do rio Tibagi (círculos escuros), rio Iguaçu (quadrados claros) e Lagoa dos Quadros (triângulos escuros) nos primeiro e segundo eixos canônicos.

interorbital. Em Lagoa dos Quadros o comprimento do barbilhão maxilar e a largura da cintura escapular foram maiores que nas outras populações (Tab. III).

\section{DISCUSSÃO}

As populações de Corydoras paleatus analisadas apresentaram variações morfométricas importantes para a sua identificação. Estas diferenças foram observadas com as análises multivariadas, que são mais recomendadas do que as análises unidimencionais, tais como as de índices ou de proporções corporais, pois permitem a interpretação da forma independente do tamanho (ReIs et al. 1987). Entretanto, estas técnicas que simplesmente removem as informações sobre o tamanho são úteis para classificar grupos de organismos, mas não para determinar o fenômeno biológico que leva a esta diferenciação (Bookstein et al. 1985). Strauss (1985) recomendava estudos ontogenéticos, como o exame da alometria para se conhecer os padrões de variação intra e interespecíficas em populações de Corydoras geograficamente isoladas. A variação ontogenética dos caracteres em $C$. paleatus deste estudo evidenciou um padrão de crescimento particular em cada população.

A discriminação da população do Iguaçu pelo primeiro eixo da variável canônica e pelo maior número de placas laterais pode ser interpretada como forte evidência do isolamento geográfico dessa população. Provavelmente a presença de $C$. 
Tabela III. Autovalores e probabilidades associadas ( $P$ ) aos primeiro e segundo eixos da análise das variáveis canônicas das amostras combinadas de Corydoras paleatus do rio Tibagi, rio Iguaçu e Lagoa dos Quadros.

\begin{tabular}{lcccc}
\hline & CAN1 & $P$ & CAN2 & $P$ \\
\hline CP & 0,22049 & 0,0933 & 0,26288 & 0,0443 \\
CC & 0,48385 & 0,0001 & 0,07792 & 0,5575 \\
DPD & 0,27425 & 0,0356 & $-0,03165$ & 0,8119 \\
CBD & 0,51009 & 0,0001 & 0,14423 & 0,2758 \\
DPV & 0,22859 & 0,0816 & 0,71010 & 0,0001 \\
CBA & $-0,37636$ & 0,0033 & 0,03365 & 0,8002 \\
AC & 0,25454 & 0,0517 & $-0,00579$ & 0,9653 \\
APC & $-0,54825$ & 0,0001 & $-0,13366$ & 0,3129 \\
DO & $-0,48354$ & 0,0001 & 0,11570 & 0,3829 \\
DIO & 0,21126 & 0,1082 & 0,58461 & 0,0001 \\
CF & 0,49913 & 0,0001 & 0,57275 & 0,0001 \\
CBM & 0,13926 & 0,2928 & $-0,55172$ & 0,0001 \\
ACb & $-0,21073$ & 0,1091 & $-0,06072$ & 0,6478 \\
LCE & 0,20244 & 0,1241 & $-0,37902$ & 0,0031 \\
DDAd & $-0,04976$ & 0,7082 & $-0,30680$ & 0,0181 \\
AAED & 0,14756 & 0,2647 & $-0,07380$ & 0,5785 \\
\hline
\end{tabular}

paleatus no rio Iguaçu seja natural e não uma introdução pelo homem, como se supunha (GARAVELlo et al. 1997), pois a região está incluída nos limites de distribuição da espécie. É possível que a espécie não tenha sido coletada por Haseman em dezembro de 1908 e dezembro de 1909, pois não foi mencionada nos primeiros trabalhos com peixes do rio Iguaçu realizados a partir daquela coleção (Haseman 1911a, b, Ellis 1911, Eigenmann 1911, 1921, 1927). Ela foi registrada 70 anos depois, por Godoy (1979), como ocorreu com outras espécies (como pode ser verificado em Garavello et al. 1997). Se a presença desta espécie é natural na bacia, seu isolamento ocorreu, no máximo, há 22 m.a. quando as cataratas foram formadas (SEVERI \& CORDEIRo 1994). O alto número de espécies endêmicas no rio Iguaçu certamente é conseqüência do evento de separação das populações deste rio das do rio Paraná, mas prováveis intercâmbios de ictiofauna entre as cabeceiras dos rios Iguaçu, Tibagi e também do Ribeira de Iguape, talvez possam ser demonstrados após um completo inventário das espécies e de suas análises filogenéticas.

Outra espécie de peixe suspeita de ter sido introduzida no rio Iguaçu é Hoplias malabaricus (Bloch, 1794). Dergam et al. (1998), demonstraram similaridade genética entre a população do rio Iguaçu com a população de cabeceira do rio Tibagi usando a técnica de estudo do polimorfismo do DNA amplificado ao acaso (RAPD). Esta técnica seria extremamente recomendável em complementação aos estudos da população de C. paleatus do rio Iguaçu. Entretanto, a análise das variáveis canônicas (AVC) foi suficiente para diferenciar a população de C. paleatus do rio Tibagi da bacia do Iguaçu.
Este é o primeiro registro desta espécie na bacia do rio Tibagi, a qual se destaca por ser o extremo norte de sua distribuição. É importante ressaltar que o rio Tibagi deságua no rio Paranapanema, mas nenhum exemplar foi coletado neste rio (CAstro et al. 2003, O.A. Shibatta, obs. pessoal).

A terceira população, Lagoa dos Quadros, também pôde ser distinguida pela AVC. De acordo com Beurlen (1970), as lagoas costeiras do Rio Grande do Sul, das quais a Lagoa dos Quadros faz parte, foram formadas no Quaternário e é provável que a população analisada foi isolada neste período das bacias adjacentes.

A hipótese da existência de várias espécies crípticas sob o nome $C$. paleatus não pode ser descartada. Esta possibilidade poderia ser refutada se um padrão morfométrico clinal fosse demonstrado com a inclusão de mais amostras representativas e de distribuição intermediária. Além disso, a união de análises morfométricas e genéticas nas amostras de diferentes localidades irá certamente permitir um panorama mais detalhado da variação geográfica desta espécie.

Evidências citogenéticas apresentadas por Oliveira et al. (1993) são corroboradas neste trabalho. Esses autores demonstraram diferenças citogenéticas nos números de pares de Regiões Organizadoras de Nucléolos entre as populações do Iguaçu e Rio Grande do Sul (São Leopoldo, bacia do Jacuí e Rio Grande, bacia da Lagoa dos Patos) apesar do número diplóide ter sido o mesmo.

As diferenças morfométricas e citogenéticas, aliadas ao isolamento geográfico, são suficientes para considerar que cada população já está sujeita a diferentes pressões seletivas. As variações intraespecíficas observadas evidenciam que análises morfométricas populacionais podem ser úteis no estudo da diversidade biológica de determinada região. Segundo GASTON \& SPICER (1998), a população é um dos elementos da biodiversidade que reflete tanto a diversidade de organismos, quanto a genética e a ecológica.

\section{AGRADECIMENTOS}

Agradecemos a Claudio de Oliveira (UNESP-Botucatu), Marcelo R. Britto (MNRJ) e Roberto E. Reis (MCP/PUCRS) pela leitura do manuscrito e valiosas sugestões. A Claudio de Oliveira, Roberto E. Reis (MCP/PUCRS), Harumi Suzuki (NUPELIA) e Vinicius Abilhoa (MCI), pelo empréstimo de material. À Cláudia B. R. Martinez pela correção do abstract. À UEL pelo suporte logístico e à FINEP pelo suporte financeiro ao projeto "Aspectos da Fauna e Flora da Bacia do rio Tibagi” (Proc. 1832/95).

\section{REFERÊNCIAS}

Beurlen, K. 1970. Geologie von brasilien. Berlin, Gebrüder Borntraeder, 1144p.

Bookstein, F.L.; B. Chernoff; R. Elder; J. Humphries; G. Smith \& R. Strauss. 1985. Morphometrics in Evolutionary Biology. Philadelphia, The Academy of Natural Sciences of Philadelphia, Special Publication no. 15, 277p

Revista Brasileira de Zoologia 22 (2): 366-371, junho 2005 
BurgEss, W.E. 1989. An atlas of freshwater and marine catfishes: a preliminary survey of the Siluriformes. Neptune City, T.F.H., 784p.

Castro, R.M.C.; L. Casatti; H.F. Santos; K.M. Ferreira; A.C. RibeiRO; R.C. Benine; G.Z.P. Dardis; A.L.A. Melo; R. Stopiglia; T.X. Abreu; F.A. Bockmann; M. Carvalho; F.Z. Gibran \& F.C.T. Lima. 2003. Estrutura e composição da ictiofauna de riachos do rio Paranapanema, Sudeste e Sul do Brasil. Biota Neotropica [online], vol.3, no. 1, p. 1-31. Available from World Wide Web: http://www.biotaneotropica.org.br/v3n1/pt/abstract? article+BN01703012003.

Dergam, J.; H.I. Suzuki; O.A. Shibatta; L.F. Duboc; H.F. Júlio-Jr.; L. Giuliano-Caetano \& W.C. Black IV. 1998. Molecular biogeography of the neotropical fish Hoplias malabaricus (Erythrinidae: Characiformes) in the Iguaçu, Tibagi, and Paraná Rivers. Genetics and Molecular Biology, Ribeirão Preto, 21 (4): 493-496.

Eigenmann, C.H. 1911. New characins in the collection of Carnegie Museum. Annals of the Carnegie Museum, Pittsburgh, 8 (1): 164-181.

Eigenmann, C.H. 1921. The American Characidae. Memoirs of the Museum of Comparative Zoology (Harvard College), Cambridge, 43 (3): 209-310.

Eigenmann, C.H. 1927. The American Characidae. Memoirs of the Museum of Comparative Zoology (Harvard College), Cambridge, 43 (4): 311-428.

Ellis, M.D. 1911. On the species of Hasemania, Hyphessobrycon and Hemigrammus collected by D.Haseman for the Carnegie Museum. Annals of the Carnegie Museum, Pittsburgh, 8 (1): 148-163.

Fowler, H.W. 1954. Os peixes de água doce do Brasil. São Paulo, Arquivos de Zoologia do estado de São Paulo, Departamento de Zoologia da Secretaria da Agricultura, vol. 9, 400p.

Garavello, J.C.; C.S. Pavanelli \& H.I. Suzuki. 1997. Caracterização da ictiofauna do rio Iguaçu, p. 61-84. In: A.A. AgostinHo \& L.C. GoMEs (Eds). Reservatório de Segredo: bases ecológicas para o manejo. Maringá, Editora da Universidade Estadual de Maringá, 387p.

GASTON, K.J. \& J.I. SPICER. 1998. Biodiversity, an introduction. Oxford, Blackwell Sciences, 113p.

Godoy, M.P. 1979. Rio Iguaçu, Paraná, Brasil. Reconhecimen- to da ictiofauna, modificações ambientais e usos múltiplos dos reservatórios. Eletrosul, Rio de Janeiro, 33p.

HaSEMAn, J.D. 1911a. An annotated catalog of the cichlid fisjhes collected by the Expedition of the Carnegie Museum of Central South America, 1907-10. Annals of the Carnegie Museum, Pittsburgh, 7 (3-4): 329-373.

Haseman, J.D. 1911b. Some new species of fishes from the Rio Iguassu. Annals of the Carnegie Museum, Pittsburgh, 7 (3-4): 374-387.

JOLICOUER, P. 1963. The multivariate generalizations of the allometry equation. Biometrics, Arlington, 19: 497-499.

MacLeod, N. 1990. Shear, p. 5. In: F.J. RohlF \& F.L. Bookstein (Eds). Proceedings of Michigan Morphometrics Workshop. University of Michigan, Museum of Zoology, 380p.

NefF, N.A. \& L.F. Marcus. 1980. A survey of multivariate methods for Systematics. New York, Privately Published, 235p.

Oliveira, C.; L.F. Almeida-Toledo; L. Mori \&S.A. Toledo-Filho. 1993. Cytogenetic and DNA contents studies of armored catfishes of the genus Corydoras (Pisces, Siluriformes, Callichthyidae) from the southeast coast of Brazil. Revista Brasileira de Genética, Ribeirão Preto, 16 (3): 617-629.

ReIs, R.E. 2003. Family Callichthyidae (Armored catfishes), p. 291-309. In: R.E. Reis; S.O. Kullander \& C.J. Ferraris (Eds.). Check list of freshwater fishes of South and Central América. Porto Alegre, EDIPUCRS, 729p.

Reis, S.F.; R.A. Cunha; J.C. Garavello \& A.S. Abe. 1987. Discriminação pela forma em relação ao tamanho: um exemplo com peixes do gênero Leporinus. Ciência e Cultura, São Paulo, 39 (8): 757-761.

Reis, S.F.; L.M. Pessôa \& R.E. Strauss. 1990. Application of sizefree canonical discriminant analysis to studies of geographical differentiation. Revista Brasileira de Genética, Ribeirão Preto, 13 (3): 509-520.

SAS Institute INC. 1985. SAS User's guide: statistics, version 5 Edition. Cary, NCSAS Institute Inc.

Severi, W. \& A.A.M Cordeiro. 1994. Catálogo de peixes da bacia do rio Iguaçu. Curitiba, IAP/GTZ, 128p.

STRAUSS, R.E. 1985. Evolutionary allometry and variation in body form in the South American catfish genus Corydoras (Callichthyidae). Systematics Zoology, Philadelphia, 34: 381-396.

Recebido em 05.VIII.2004; aceito em 13.V.2005. 